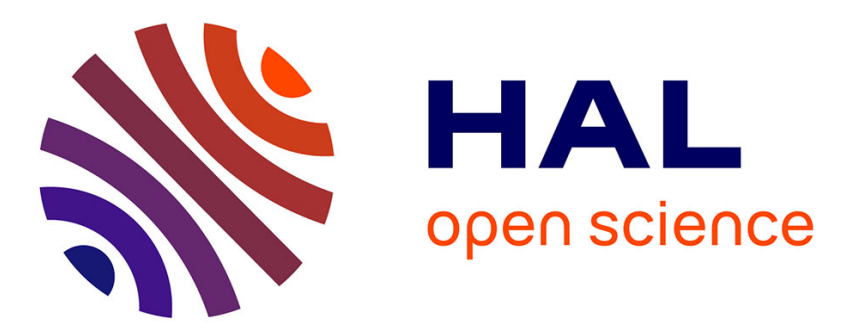

\title{
Frustration enhanced by Kitaev exchange in a jeff $=12$ triangular antiferromagnet
}

C Wellm, W Roscher, J Zeisner, A Alfonsov, R Zhong, R Cava, Adrien Savoyant, R Hayn, J van den Brink, B Büchner, et al.

\section{To cite this version:}

C Wellm, W Roscher, J Zeisner, A Alfonsov, R Zhong, et al.. Frustration enhanced by Kitaev exchange in a jeff=12 triangular antiferromagnet. Physical Review B, 2021, 104, 10.1103/physrevb.104.1100420 . hal-03586808

\section{HAL Id: hal-03586808 \\ https://hal.science/hal-03586808}

Submitted on 24 Feb 2022

HAL is a multi-disciplinary open access archive for the deposit and dissemination of scientific research documents, whether they are published or not. The documents may come from teaching and research institutions in France or abroad, or from public or private research centers.
L'archive ouverte pluridisciplinaire HAL, est destinée au dépôt et à la diffusion de documents scientifiques de niveau recherche, publiés ou non, émanant des établissements d'enseignement et de recherche français ou étrangers, des laboratoires publics ou privés. 


\title{
Frustration enhanced by Kitaev exchange in a $\tilde{j}_{\text {eff }}=\frac{1}{2}$ triangular antiferromagnet
}

\author{
C. Wellm $\odot,{ }^{1,2}$ W. Roscher $\odot,{ }^{1}$ J. Zeisner, ${ }^{1,2}$ A. Alfonsov $\odot,{ }^{1}$ R. Zhong, ${ }^{3}$ R. J. Cava, ${ }^{3}$ A. Savoyant $\odot,{ }^{4}$ R. Hayn $\odot,{ }^{1,4}$ \\ J. van den Brink, ${ }^{1,5}$ B. Büchner, ${ }^{1,5}$ O. Janson ${ }^{1},{ }^{1}$ and V. Kataev (1) ${ }^{1}$ \\ ${ }^{1}$ Leibniz Institute for Solid State and Materials Research, IFW Dresden, 01171 Dresden, Germany \\ ${ }^{2}$ Institute for Solid State and Materials Physics, Technische Universität Dresden, 01069 Dresden, Germany \\ ${ }^{3}$ Department of Chemistry, Princeton University, Princeton, New Jersey 08544, USA \\ ${ }^{4}$ Aix-Marseille Université, Centre National de la Recherche Scientifique, IM2NP-UMR 7334, 13397 Marseille Cedex 20, France \\ ${ }^{5}$ Würzburg-Dresden Cluster of Excellence ct.qmat, Technische Universität Dresden, D-01062 Dresden, Germany
}

(Received 21 December 2020; revised 1 August 2021; accepted 15 September 2021; published 30 September 2021)

\begin{abstract}
Triangular Heisenberg antiferromagnets are prototypes of geometric frustration, even if for nearest-neighbor interactions quantum fluctuations are not usually strong enough to destroy magnetic ordering: stronger frustration is required to stabilize a spin-liquid phase. On the basis of static magnetization and electron spin resonance measurements, we demonstrate the emergence of $\tilde{j}_{\text {eff }}=\frac{1}{2}$ moments in the triangular-lattice magnet $\mathrm{Na}_{2} \mathrm{BaCo}\left(\mathrm{PO}_{4}\right)_{2}$. These moments are subject to an extra source of frustration that causes magnetic correlations to set in far above both the magnetic ordering and Weiss temperatures. Corroborating the $\tilde{j}_{\text {eff }}=\frac{1}{2}$ ground state, theory identifies ferromagnetic Kitaev exchange anisotropy as an additional frustrating agent, altogether putting forward $\mathrm{Na}_{2} \mathrm{BaCo}\left(\mathrm{PO}_{4}\right)_{2}$ as a promising Kitaev spin-liquid material.
\end{abstract}

DOI: 10.1103/PhysRevB.104.L100420

\section{INTRODUCTION}

Quantum spin liquids are of topical interest as a promising host for fractionalized quasiparticles [1-5]. The quest for a spin-liquid ground state goes back to Anderson's conjecture on resonating valence bonds in the geometrically frustrated two-dimensional triangular lattice [6]. It is now firmly established, however, that the pure spin $1 / 2$ triangularlattice Heisenberg model has an ordered ground state [7]. This seemingly frustrating result is a blessing in disguise, though, as subsequent studies of modified triangular-lattice models found that long-range ordering can be suppressed by three disparate alterations: additional second-neighbor exchange [8-13], spatial anisotropy [14,15], and exchange anisotropy [16,17]. These findings opened new perspectives for realizing a spin-liquid state in the triangular lattice geometry, and boosted the search for candidate materials.

A recent breakthrough in triangular-lattice magnets pertains to the discovery of two material classes [18]. The first group of candidate materials are $4 f$ magnets with $\mathrm{Yb}^{3+}$ : $\mathrm{YbMgGaO}_{4}[19,20]$ and $\mathrm{NaYb}_{2}$ (with $X=\mathrm{O}, \mathrm{S}, \mathrm{Se}$ ) [21]. The key element of their physics is the crystal-field (CF) splitting of $\mathrm{Yb}^{3+}$, giving rise to the ground-state doublet and hence the effective spin $1 / 2$ behavior at low temperatures, and, in the former material, the significant effects of chemical disorder between the magnetic layers [22]. A similar scenario, with the lowest-lying doublet dominating the low-temperature physics, is realized in the other promising group of materials- $\mathrm{Co}^{2+}$ cobaltates. Here, the ground-state doublet is stabilized by the spin-orbit (SO) coupling as long as the low-symmetry crystalfield distortions remain small [Fig. 1(a)]. Despite the overall tendency towards ferromagnetic exchange, several cobaltates with antiferromagnetic (AFM) interactions are known.
The most studied material of this handful, $\mathrm{Ba}_{3} \mathrm{CoSb}_{2} \mathrm{O}_{9}$, shows intriguing high-field behavior [23-25], but evades the spin-liquid regime by developing a long-range order at 3.7 K [23]. Interestingly, in the related layered honeycomb magnet $\mathrm{BaCo}_{2}\left(\mathrm{AsO}_{4}\right)_{2}$ the magnetic order can be suppressed by a low magnetic field yielding a nonmagnetic material [26].

The recently synthesized cobaltate $\mathrm{Na}_{2} \mathrm{BaCo}\left(\mathrm{PO}_{4}\right)_{2}$ [Fig. 1(b)] is a promising candidate for realization of a spin-liquid state on a triangular lattice. The first experimental study reported a sizable antiferromagnetic exchange and no long-range ordering down to $50 \mathrm{mK}$ [27]. Recently, ultralow-temperature specific heat measurements revealed a magnetic ordering transition at $T_{\mathrm{N}}=148 \mathrm{mK}$ [28], i.e., $\approx 20$ times smaller than the Weiss temperature $\theta=2.5 \mathrm{~K}$ obtained from the static magnetic susceptibility $\chi(T)$ [28]. Another interesting observation is the linear dependence of the residual thermal conductivity [28], compatible with the spinon Fermi surface scenario advocated for critical spin liquids. Indications for the spinonlike excitations were also found in recent nuclear magnetic resonance experiments [29]. However, the microscopic model for $\mathrm{Na}_{2} \mathrm{BaCo}\left(\mathrm{PO}_{4}\right)_{2}$ remains hitherto unexplored.

In this Letter, we fill this gap and demonstrate that exchange anisotropy stabilizes the spin-liquid behavior in $\mathrm{Na}_{2} \mathrm{BaCo}\left(\mathrm{PO}_{4}\right)_{2}$ over a temperature range spanning two orders of magnitude. Our $\mathrm{Co}^{2+}$ electron spin resonance (ESR) measurements firmly establish the effective spin $1 / 2$ state of the $\mathrm{Co}^{2+}$ ions [30] by determining the $g$ factor tensor and evaluating the excitation energy of the first excited state with effective spin $3 / 2$. Further, the ESR data indicate the onset of magnetic correlations at the surprisingly high temperature of $\approx 20 \mathrm{~K}$, which is two orders of magnitude larger than $T_{\mathrm{N}}$. Hence, magnetic correlations in this 
(a)

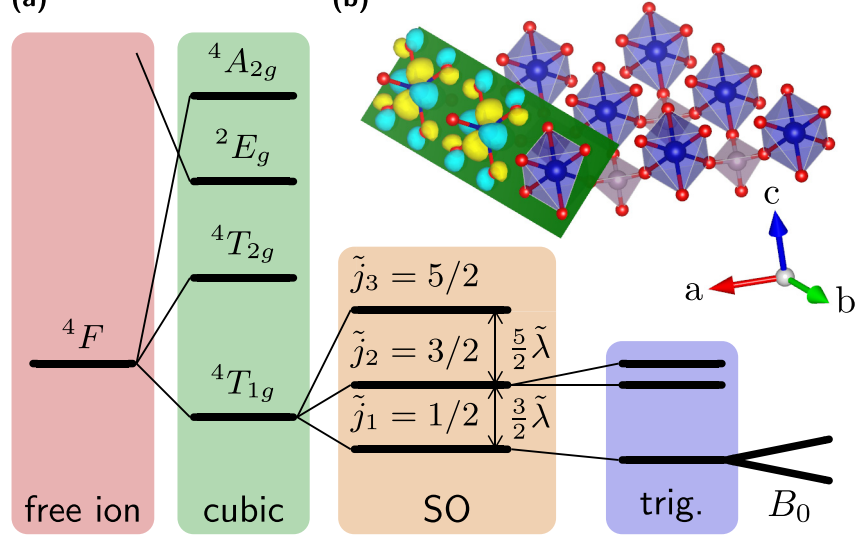

FIG. 1. (a) Energy diagram showing the splitting of the ${ }^{4} F$ state of $\mathrm{Co}^{2+}$ in a cubic crystal field, the SO coupling, trigonal distortion, and the Zeeman splitting due to the magnetic field $B_{0}$ in the ${ }^{4} T_{1 g}$ multiplet. (b) Fragment of a triangular layer in the $\mathrm{Na}_{2} \mathrm{BaCo}\left(\mathrm{PO}_{4}\right)_{2}$ crystal structure, with $\mathrm{CoO}_{6}$ octahedra bridged by $\mathrm{PO}_{4}$ tetrahedra. For a pair of neighboring Co atoms, the leading contribution to hopping stems from the depicted Wannier functions. Note that the respective Co $d$ orbitals lie in the same plane.

material build up far above the phenomenological energy scale set by the moderate Weiss temperature and the low saturation field of the magnetization. To reconcile these seemingly conflicting observations, we perform a microscopic analysis based on density-functional-theory (DFT) band-structure and multiplet calculations. We confirm the effective spin $1 / 2$ (hereafter $\tilde{j}_{\text {eff }}=\frac{1}{2}$ ) behavior, estimate theoretically the $g$ factors, and justify the restriction of the magnetic system to the strong dominance of the nearest-neighbor exchange $J_{1}$, which however turns out to be substantially larger than previous experimental estimates. By applying perturbation-theory expressions from Refs. [31,32] to the multiorbital Hubbard model parametrized by DFT calculations, we conclude that $\mathrm{Na}_{2} \mathrm{BaCo}\left(\mathrm{PO}_{4}\right)_{2}$ features a substantial ferromagnetic Kitaev exchange $K_{1}$. The competition of antiferromagnetic $J_{1}$ and ferromagnetic $K_{1}$ lowers the saturation field and the Weiss temperature, but promotes the buildup of magnetic correlations to higher temperatures.

\section{EXPERIMENTAL RESULTS}

Magnetization and ESR experiments were conducted on both powder and single-crystal samples of $\mathrm{Na}_{2} \mathrm{BaCo}\left(\mathrm{PO}_{4}\right)_{2}$ synthesized and thoroughly characterized in Ref. [27]. Representative magnetization $M(H)$ and the inverse susceptibility $\chi^{-1}(T)=H / M(T)$ curves are shown in Fig. 2.

The $M(H)$ dependence can be well fitted to the equation $M=g \tilde{j}_{\text {eff }} B_{1 / 2}\left(\left(H_{0}+H_{\mathrm{ex}}\right), T\right)+\chi_{0} H_{0}$. Here, $B_{1 / 2}$ is the Brillouin function for spin $1 / 2, g$ is the $g$ factor, $H_{0}$ is the external magnetic field, $\chi_{0}$ accounts for the van Vleck and diamagnetic susceptibility, and $H_{\mathrm{ex}}=a_{\mathrm{ex}} M$ is the exchange field with the parameter $a_{\mathrm{ex}}$ characterizing the strength of the isotropic exchange interaction $J=a_{\mathrm{ex}} g^{2} \mu_{\mathrm{B}} / N_{\mathrm{NN}}$ with $N_{\mathrm{NN}}$ being the number of next neighbors. A representative fit for the powder sample is shown in the main panel of Fig. 2(a).
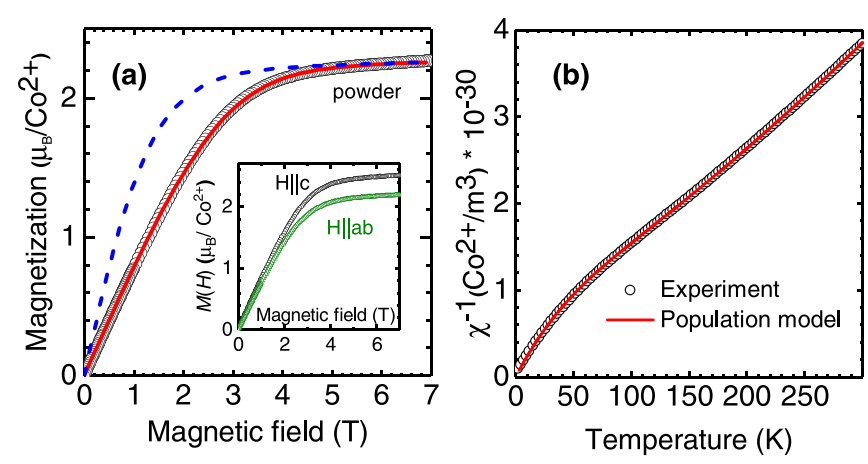

FIG. 2. (a) Main panel: Powder $M(H)$ dependence at $T=2 \mathrm{~K}$ (data points). A delayed increase of the $M(H)$ curve compared to the Brillouin function of noninteracting spins $S=1 / 2$ (dashed curve) indicates a sizable AFM interaction. Accounting for it, as explained in the text, yields a good agreement with the experiment (red solid curve). Inset: Single-crystalline $M(H)$ dependence for the $\mathbf{H} \| c$ axis and $\mathbf{H} \| a b$ plane. (b) Powder $\chi^{-1}(T)$ dependence at $\mu_{0} H=20 \mathrm{mT}$ (data points). The solid line depicts the calculated curve according to the population model (see the text).

It yields AFM $J=1.37 \mathrm{~K}$ and a $g$ factor $g_{\mathrm{P}}^{M}=4.38$. For the single crystal, the $g$ factors were found to be $g_{\mathrm{c}}^{M}=4.81$ and $g_{\mathrm{ab}}^{M}=4.22$. The slight $g$ factor anisotropy is likely related to a small trigonal distortion of the $\mathrm{CoO}_{6}$ octahedra [33].

The inverse susceptibility $\chi^{-1}(T)$ per $\mathrm{Co}^{2+}$ ion at $\mu_{0} H=20 \mathrm{mT}$ is shown in Fig. 2(b) together with the result of a population model. The latter is derived from the diagonalization of a single-ion Hamiltonian including the spin-orbit coupling and the Zeeman interactions, $\mathcal{H}=$ $\widetilde{\lambda} \mathbf{S L}-\mu_{\mathrm{B}} H\left(g_{\mathrm{S}} S_{\mathrm{Z}}+g_{\mathrm{L}} L_{\mathrm{Z}}\right)$ with $g_{\mathrm{S}}=2, g_{\mathrm{L}}=-3 / 2$ and thermal average $<M>=\mathcal{Z}^{-1} \sum_{i}\left\langle E_{\mathrm{i}}\left|e^{-E_{\mathrm{i}} / k_{\mathrm{B}} T} \mu_{\mathrm{z}}\right| E_{\mathrm{i}}\right\rangle, \mathcal{Z}$ being the partition sum. $\chi^{-1}(T)$ does not obey a simple Curie-Weiss law $\propto T$, but rather possesses a remarkable inflection point around $T \sim 150 \mathrm{~K}$ which is reasonable to attribute to the thermally assisted population of the excited $\tilde{j}_{2}=\frac{3}{2}$ multiplet (Fig. 1). The modeling reproduces the experimental data with the energy splitting $\Delta E_{\tilde{j}_{1}, \tilde{j}_{2}}=E_{\tilde{j}_{2}}-E_{\tilde{j}_{1}}=419 \mathrm{~K}$.

The main results of the ESR measurements for both the powder- and single-crystalline samples at the X-band frequency of $v=9.6 \mathrm{GHz}$ conducted with a commercial ESR spectrometer from Bruker are summarized in Figs. 3(a) and 3(b). A rather broad Lorentzian-shaped signal was observed [Fig. 3(a)]. The Lorentzian fitting yielded the width $\Delta H$ and the resonance field $H_{\text {res }}$ from which the effective $g$ factor can be calculated according to resonance condition $h v=g \mu_{\mathrm{B}} H_{\mathrm{res}}$.

The angular dependent measurements revealed an anisotropy of the $g$ factor which follows the conventional angular dependence $g(\alpha)=\sqrt{g_{\mathrm{ab}}^{2} \cos ^{2}(\alpha)+g_{\mathrm{c}}^{2} \sin ^{2}(\alpha)}$ with $\alpha$ being the angle which the applied field makes with the $c$ axis [Fig. 3(a), inset]. The fit yielded the values $g_{\mathrm{c}} \approx 4.83$ and $g_{\mathrm{ab}} \approx 4.42$ for the out-of-plane and in-plane orientation of the field, respectively, consistent with the static magnetization data.

More accurate estimates of the $g_{\mathrm{ab}}$ value were provided by multifrequency high-field ESR (HF-ESR) measurements on the powder sample which were carried out with a 

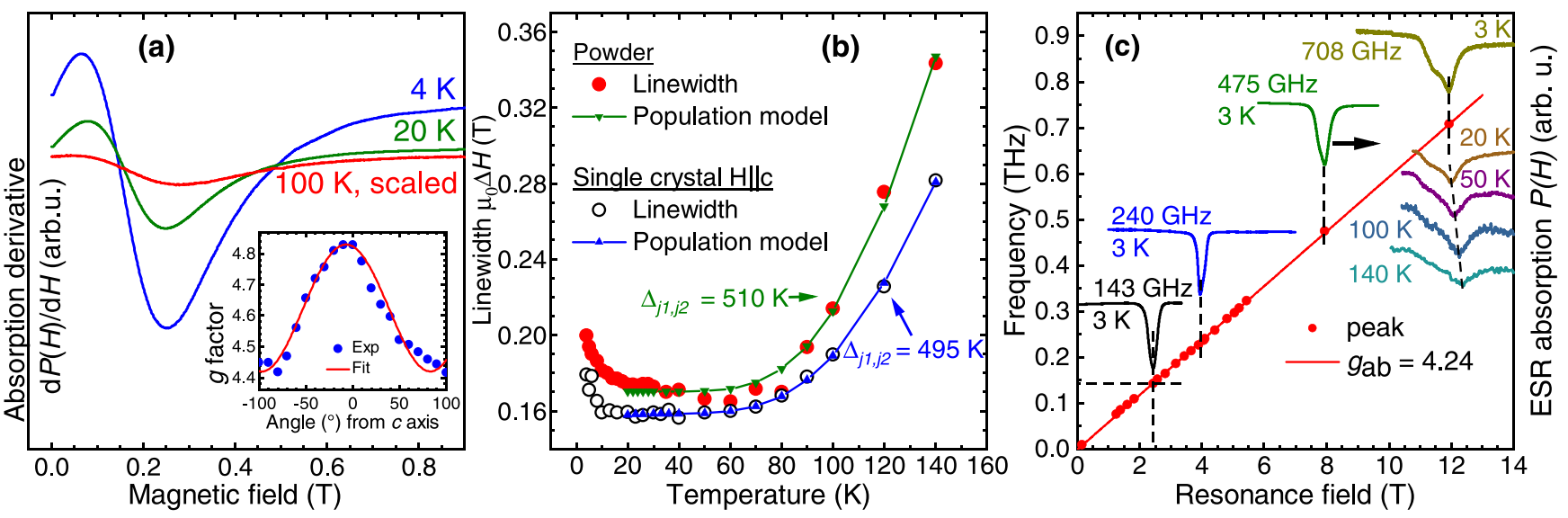

FIG. 3. (a) Single-crystalline ESR signals (field derivative of the microwave absorption) at $v=9.6 \mathrm{GHz}$ for the $\mathbf{H} \| c$ axis at selected temperatures. Inset: Angular dependence of the $g$ factor at $v=9.6 \mathrm{GHz}$ and at $T=4 \mathrm{~K}$ (data points). The solid line is a fit to the dependence $g(\alpha)=\left[g_{\mathrm{ab}}^{2} \cos ^{2}(\alpha)+g_{\mathrm{c}}^{2} \sin ^{2}(\alpha)\right]^{1 / 2}$. (b) $T$ dependence of the ESR linewidth of the single- and polycrystalline samples at $v=9.6 \mathrm{GHz}($ data points). Solid lines are the calculated dependences according to the population model (see the text). (c) Left scale: $v$ vs field position of the peak of the powder HF-ESR spectrum at $T=3 \mathrm{~K}$ (dots). The solid line is the fit to the dependence $h v=g \mu_{\mathrm{B}} H$. Right scale: Powder HF-ESR spectra at selected frequencies $v$ at $T=3 \mathrm{~K}$ and at selected temperatures at $v=708 \mathrm{GHz}$.

homemade setup [34]. Selected HF-ESR spectra at $T=3 \mathrm{~K}$ and different excitation frequencies are shown in Fig. 3(c). The signal is a single Lorentzian-shaped line at lower frequencies and develops a shoulder at $v \geqslant 150 \mathrm{GHz}$. Such a structure typical of the ESR response of a powder sample with an anisotropic $g$ factor [35] can be most clearly resolved at the highest frequency of $708 \mathrm{GHz}$. In the case of the uniaxial $g$ anisotropy the resonance field of the peak is determined by the $g$ factor component perpendicular to the symmetry axis, i.e., by $g_{\text {ab }}$ in our case [35]. The field position of the peak plotted versus $v$ exhibits a linear dependence according to the relation $h v=g_{\mathrm{ab}} \mu_{\mathrm{B}} H$ and gives $g_{\mathrm{ab}}^{\mathrm{HF}}=4.24$ [Fig. 3(c)]. Note that this linear dependence features a negligible intercept with the frequency axis evidencing the absence of any gap in the uniform spin excitation spectrum larger than $9.6 \mathrm{GHz}(0.04 \mathrm{meV})$. Thus, taken together the X-band and HF-ESR results yield the g tensor $\left[g_{\mathrm{c}}, g_{\mathrm{ab}}\right]=[4.83,4.24]$ consistent with the estimate $\left[g_{\mathrm{c}}^{M}, g_{\mathrm{ab}}^{M}\right]=[4.81,4.22]$ from the magnetization data. Interestingly, with increasing the temperature close to $100 \mathrm{~K}$ the peak of the HF-ESR spectrum at $v=708 \mathrm{GHz}$ shifts by an amount of $\mu_{0} \delta H_{0} \approx 0.35 \mathrm{~T}$ towards higher fields, suggesting a reduction of the $g$ factor [Fig. 3(c)]. At $T=100 \mathrm{~K}$, a linear $v$ versus $H g$ factor fit of this peak yields $g_{\mathrm{c}}(100 \mathrm{~K})=4.11$. Such a reduction indicates the involvement of the thermally populated excited $\tilde{j}_{2}=\frac{3}{2}$ multiplet which is characterized by a smaller $g$ factor [33].

Both X-band and HF-ESR spectra broaden significantly with increasing the temperature [Figs. 3(a) and 3(c)]. Analysis of the origin of this broadening provides important insights into the spin dynamics and temperature effects in $\mathrm{Na}_{2} \mathrm{BaCo}\left(\mathrm{PO}_{4}\right)_{2}$. On the quantitative level, the $\Delta H(T)$ dependence can be more accurately evaluated from the X-band data because strong fields applied in an HF-ESR experiment may cause additional inhomogeneous broadening, and instrumental distortions of the line shape are possible in the HF-ESR apparatus.

In Fig. 3(b), the temperature dependence of the linewidth, as obtained from the Lorentzian fits, is shown for the powder- and single-crystalline samples. While lowering the temperature the width decreases down to approximately $50 \mathrm{~K}$, then remains constant until approximately $20 \mathrm{~K}$, where it starts to rise again. The high-temperature behavior is likely due to the above discussed population effect of the excited $\tilde{j}_{2}=\frac{3}{2}$ multiplet [Fig. 1(a)]. This leads to a broadening of the linewidth, while at lower temperatures, only the $\tilde{j}_{1}=\frac{1}{2}$ multiplet is populated. This behavior can be reproduced by a population model with Boltzmann weights for the energy levels of the $\mathrm{Co}^{2+}$ ions in an octahedral oxygen environment, as described in Ref. [36]. From this model, one obtains the energy splitting $\Delta E_{\tilde{j}_{1}, \tilde{j}_{2}}^{\text {pow }}=510 \mathrm{~K}$ for the powder sample, which is somewhat larger than the estimate from the modeling of the static susceptibility. For the single crystal, one has, similarly, $\Delta E_{\tilde{j}_{1}, \tilde{j}_{2}}^{\mathrm{cr}}=495 \mathrm{~K}$. For the in-plane direction of the field, the linewidth could not be modeled well due to the uncertainty of the Lorentzian fits.

The upturn of $\Delta H(T)$ below $20 \mathrm{~K}$ is remarkable. It cannot be described by the single-ion population model, suggesting that magnetic correlations between the Co spins begin to develop gradually. Usually this signifies proximity to a magnetic phase transition where the spin dynamics experiences a critical slowing down [37]. However, such a phase transition in $\mathrm{Na}_{2} \mathrm{BaCo}\left(\mathrm{PO}_{4}\right)_{2}$ takes place at two orders of magnitude lower temperature of $T_{\mathrm{N}}=148 \mathrm{mK}$ [28] which evidences an extended low-temperature regime of the spin-liquid-like, slow spin fluctuations in the electron spin system.

\section{THEORY AND DISCUSSION}

To understand why magnetic correlations set on at such a high temperature, we performed a microscopic numerical analysis based on DFT band-structure as well as multiplet calculations. For the former, we do full-relativistic nonmagnetic DFT calculations within the generalized gradient approximation (GGA) [38], as implemented in the full-potential local-orbital code FPLO [39]. As an input, we use the experimental crystal structure described within the space group 


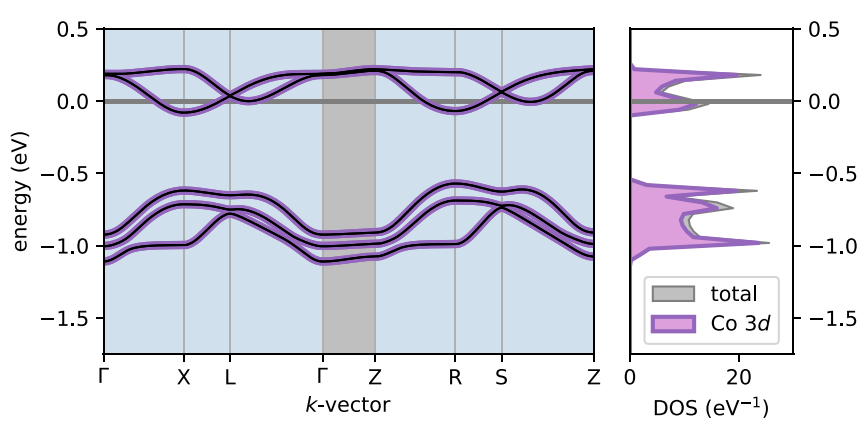

FIG. 4. Left: Full relativistic GGA band structure (thin black curves) and the eigenvalues of the Fourier-transformed Wannier Hamiltonian (thick violet curves). All bands are doubly degenerate due to the time-reversal symmetry. Minute dispersions along $\Gamma-Z$ are indicative of a quasi-two-dimensional electronic structure. Right: Total density of states (DOS) and Co $3 d$ contributions. The Fermi level is at zero energy.

$P \overline{3} m 1$ [27]. The unit cell contains one Co atom with the local trigonal symmetry (point group $\overline{3} m$ ). The GGA band structure features a ten-band manifold crossing the Fermi level, as expected for a unit cell containing one Co atom featuring five $d$ orbitals and two spin flavors. In the absence of spin polarization, each band is doubly degenerate in accord with the Kramers theorem. As a result, the band structure features only five distinct band dispersions (Fig. 4, left). The strong octahedral crystal field splits these bands into two manifolds that are well separated from the rest of the valence band and are formed almost exclusively by Co $d$ states (Fig. 4, right) [40]. For further analysis, we single out these states by resorting to Co-centered Wannier functions (WFs). Following the procedure described in Ref. [41], we obtain excellent agreement between the Fourier-transformed WF and the respective GGA bands [Fig. 4(a)].

In the WF basis, the Hamiltonian is a sum of local and nonlocal terms. The former describes the one-particle spectrum of an isolated trigonally distorted $\mathrm{CoO}_{6}$ octahedron, and key parameters of the local Hamiltonian can be extracted directly from WF [42]. By inspecting different contributions, we find a well-defined hierarchy of energy scales: the strong cubic $\mathrm{CF}$, followed by the SO interactions, the trigonal CF splitting, and finally, the Zeeman term. The separation of energy scales allows us to disentangle the underlying processes and arrive at an intuitive physical picture, which we show in Fig 1(a). First, the cubic CF splits the ${ }^{4} F$ multiplet of the free $\mathrm{Co}^{2+}$ ion into three multiplets where the lower one, ${ }^{4} T_{1 g}$, contains predominantly only one-electron $t_{2 g}$ holes. Next, the ${ }^{4} T_{1 g}$ multiplet is further split by the SO interaction. An isolated ${ }^{4} T_{1 g}$ multiplet can be solved analytically [33], resulting in effective $\tilde{j}$ values, from lowest to highest energy $\tilde{j}_{1}=\frac{1}{2}, \tilde{j}_{2}=\frac{3}{2}$, and $\tilde{j}_{3}=\frac{5}{2}$ [43]; the respective $g$ factors amount to $g_{1}=\frac{13}{3}$, $g_{2}=\frac{16}{15}$, and $g_{3}=\frac{3}{5}$, and the two splittings amount to $\frac{3}{2} \tilde{\lambda}$ and $\frac{5}{2} \lambda$, where $\widetilde{\lambda}=33 \mathrm{meV}$ is the effective SO interaction parameter [44]. The trigonal CF further splits the second and third multiplets, giving rise to the experimentally observed $g$ factor anisotropy. Finally, the Zeeman interaction lifts the remaining degeneracy.

The GGA calculations (Fig. 4) imply a one-electron picture in an effective mean-field potential and cannot display the multiplet structure directly. To account for the ensuing electronic correlations, we perform multiplet calculations using the parameters extracted from the WF, and obtain a complete numerical solution of the local many-body problem. Skipping methodological aspects of this procedure that will be published elsewhere, we go straight to the most important result: the splitting between the $\tilde{j}_{1}=\frac{1}{2}$ ground state and the lowest-lying $\tilde{j}_{2}=\frac{3}{2}$ component amounts to $\Delta E_{\tilde{j}_{1}, \tilde{j}_{2}}=472 \mathrm{~K}$, in a fair agreement with our experimental estimates [45]. The splitting exceeds by far the magnetic energy scale. Therefore, the magnetism of $\mathrm{Na}_{2} \mathrm{BaCo}\left(\mathrm{PO}_{4}\right)_{2}$ can be safely described in an effective $\tilde{j}_{\text {eff }}=\frac{1}{2}$ model. Another important observable is the $\mathbf{g}$ tensor anisotropy, i.e., the difference between $g_{c}$ and $g_{a b}$. Our calculations lead to an anisotropy of $g_{\mathrm{c}}=4.33$ and $g_{\mathrm{ab}}=$ 4.55 being slightly smaller than the experimental one and of opposite sign. The reason for this discrepancy is the extreme sensitivity of the $g$ factor anisotropy to the small $(10 \mathrm{meV})$ trigonal crystal-field parameter: a small readjustment of the CF parameter reproduces the experimental values $\left(g_{c}{ }_{c}^{\text {adj }}=4.80\right.$ and $g_{\mathrm{ab}}^{\mathrm{adj}}=4.31$ ). And since the trigonal CF is in turn governed by the oxygen positions in the crystal structure, additional structural refinements, especially at lower temperatures, are needed for a conclusive analysis.

Having solved the local problem, we turn to hopping processes that underlie the magnetic exchange. $\mathrm{By}$ analyzing the respective WF, we find that $\mathrm{Na}_{2} \mathrm{BaCo}\left(\mathrm{PO}_{4}\right)_{2}$ is an excellent model system: further longer-range in-plane as well as interplane hoppings are orders of magnitude weaker than the leading nearest-neighbor terms. As we are dealing with a multiorbital problem, a direct determination of the magnetic exchange integrals from the hopping terms is very challenging. Therefore, we start with a qualitative analysis. By inspecting the hoppings between different orbitals, we find that for every pair of neighboring Co atoms, the leading contribution pertains to a single matrix element: virtual electron transfer between two $t_{2 g}$ orbitals lying in the same plane [e.g., the plane in Fig. 1(b)]. Although this hopping implicitly includes transfer to and between the ligand orbitals, it is effectively equivalent to the direct hopping denoted as $t^{\prime}$ in Refs. [31,32]. We note that this hopping is underlain by the perfect mutual alignment of neighboring $\mathrm{CoO}_{6}$ octahedra and may be strongly suppressed if the octahedra are tilted.

Next, we estimate the exchange integral $J_{1}$ quantitatively. To this end, we perform full-relativistic magnetic $\mathrm{DFT}+U$ calculations (see Supplemental Material [45] and, also, Refs. [46-50] therein). We find that large orbital moments, a prerequisite for the correct description of $\tilde{j}_{\text {eff }}=\frac{1}{2}$ physics, are stable only if the quantization axis coincides with the threefold rotation crystal axis. We construct three supercells and perform total-energy calculations for different collinear magnetic arrangements. By mapping the total energies onto a classical Heisenberg model with effective spins $\tilde{j}_{\text {eff }}=\frac{1}{2}$, we estimate the nearest-neighbor exchange to be $11.6 \pm 2.0 \mathrm{~K}$, where the error bars pertain to the uncertainty in choosing the $U$ value $(U=5 \mp 1 \mathrm{eV})$.

Why is the DFT $+U$ estimate for $J_{1}$ so large? To find the root cause, we first recollect that the magnetic exchange in $\mathrm{Na}_{2} \mathrm{BaCo}\left(\mathrm{PO}_{4}\right)_{2}$ is anisotropic. As the orientation of magnetic moments in $\mathrm{DFT}+U$ is fixed, such calculations provide 
only one component of the magnetic exchange, $J_{1}^{z z}$. To estimate the anisotropic terms we go a step back and apply the recently developed perturbative expressions [31,32], parametrized by our WF. Using just the dominant hopping term $t^{\prime}=82 \mathrm{meV}$ [45] we arrive at $J_{1}^{z z}=J_{1}+K_{1} / 3=2.3 \mathrm{~K}$, which is smaller than the DFT $+U$ estimate. Surprisingly, we find, besides the leading antiferromagnetic term $J_{1}=$ $(16 / 81)\left(t^{\prime}\right)^{2} / U=3.1 \mathrm{~K}$, also a sizable ferromagnetic Kitaev term $K_{1}=(-4 / 27)\left(t^{\prime}\right)^{2} / U=-2.3 \mathrm{~K}$. Other anisotropic terms are smaller, and hence nearest-neighbor exchange and Kitaev terms dominate the magnetic interactions. These results agree with our mean-field estimate of $J$ from the magnetization $M(H)$ dependence [Fig. 2(a)].

We are now in a position to combine our results into a coherent physical picture. $\mathrm{Na}_{2} \mathrm{BaCo}\left(\mathrm{PO}_{4}\right)_{2}$ is a nearest-neighbor $\tilde{j}_{\text {eff }}=\frac{1}{2}$ triangular magnet with a strongly anisotropic exchange: Its magnetism is driven by the competition of the dominant antiferromagnetic contribution with a sizable ferromagnetic Kitaev term. The latter lowers the saturation field, which otherwise would be of the order of $J_{1}$. But the energy scale of magnetic correlations is set by the absolute values of magnetic exchanges, and hence it is enhanced by $K_{1}$. Therefore, the onset of the spin-spin correlations already at $T \sim 20 \mathrm{~K} \gg T_{\mathrm{N}}$, manifested in the observed broadening of the ESR linewidth, is a combined effect of $J_{1}$ and $K_{1}$.

\section{CONCLUSIONS}

Our static magnetization and ESR results on the spin-liquid candidate material $\mathrm{Na}_{2} \mathrm{BaCo}\left(\mathrm{PO}_{4}\right)_{2}$ enable us to classify it as an anisotropic triangular magnet in the effective spin $\tilde{j}_{\text {eff }}=\frac{1}{2}$ ground state well isolated from the higher energy effective spin $\frac{3}{2}$ state. Furthermore, the ESR data provide strong indications of the magnetic correlations setting in far above the magnetic ordering temperature $T_{\mathrm{N}}$ and the Weiss temperature $\theta$. We established the anisotropic $\tilde{j}_{\text {eff }}=\frac{1}{2}$ ground state on the theory level as well and reveal, besides the antiferromagnetic isotropic nearest-neighbor exchange interaction $J_{1}$, a significant ferromagnetic Kitaev interaction term $K$, which is beneficial for the realization of a spin liquid in this compound. The competition between $J_{1}$ and $K$ reduces the temperature scale $\theta$ but does not affect magnetic correlations the energy scale of $\approx 20 \mathrm{~K} \gg T_{\mathrm{N}}$ of which is probed by ESR. Altogether our findings put forward $\mathrm{Na}_{2} \mathrm{BaCo}\left(\mathrm{PO}_{4}\right)_{2}$ as a promising Kitaev-exchange-assisted spin-liquid material and call for further extensive exploration of the exciting physics of this class of compounds.

\section{ACKNOWLEDGMENTS}

O.J. thanks Alexander Tsirlin and R.H. thanks Roman Kuzian for fruitful discussions and helpful comments. We thank Manuel Richter for useful hints on DFT $+U$ calculations and Ulrike Nitzsche for technical assistance. W.R. and O.J. were supported by the Leibniz Association through the Leibniz Competition. This work was supported in part by Deutsche Forschungsgemeinschaft Grant No. KA 1694/12-1 and by the Collaborative Research Center SFB 1143 "Correlated Magnetism: From Frustration to Topology (Project No. 247310070). The materials synthesis was supported by the Gordon and Betty Moore Foundation, EPiQS Program, Grant No. GBMF-4412.
[1] L. Balents, Nature (London) 464, 199 (2010).

[2] L. Savary and L. Balents, Rep. Prog. Phys. 80, 016502 (2016).

[3] Y. Zhou, K. Kanoda, and T.-K. Ng, Rev. Mod. Phys. 89, 025003 (2017).

[4] J. Knolle and R. Moessner, Ann. Rev. Condens. Matter Phys. 10, 451 (2019).

[5] C. Broholm, R. J. Cava, S. A. Kivelson, D. G. Nocera, M. R. Norman, and T. Senthil, Science 367, 263 (2020).

[6] P. W. Anderson, Mater. Res. Bull. 8, 153 (1973).

[7] B. Bernu, C. Lhuillier, and L. Pierre, Phys. Rev. Lett. 69, 2590 (1992); B. Bernu, P. Lecheminant, C. Lhuillier, and L. Pierre, Phys. Scr., T 49, 192 (1993); Phys. Rev. B 50, 10048 (1994); S. R. White and A. L. Chernyshev, Phys. Rev. Lett. 99, 127004 (2007).

[8] Y. Iqbal, W.-J. Hu, R. Thomale, D. Poilblanc, and F. Becca, Phys. Rev. B 93, 144411 (2016).

[9] D.-V. Bauer and J. O. Fjærestad, Phys. Rev. B 96, 165141 (2017)

[10] S. N. Saadatmand and I. P. McCulloch, Phys. Rev. B 96, 075117 (2017).

[11] S.-S. Gong, W. Zhu, J.-X. Zhu, D. N. Sheng, and K. Yang, Phys. Rev. B 96, 075116 (2017).

[12] F. Ferrari and F. Becca, Phys. Rev. X 9, 031026 (2019).

[13] S. Hu, W. Zhu, S. Eggert, and Y.-C. He, Phys. Rev. Lett. 123, 207203 (2019).
[14] S. Yunoki and S. Sorella, Phys. Rev. B 74, 014408 (2006).

[15] E. Ghorbani, L. F. Tocchio, and F. Becca, Phys. Rev. B 93, 085111 (2016).

[16] Z. Zhu, P. A. Maksimov, S. R. White, and A. L. Chernyshev, Phys. Rev. Lett. 120, 207203 (2018).

[17] P. A. Maksimov, Z. Zhu, S. R. White, and A. L. Chernyshev, Phys. Rev. X 9, 021017 (2019).

[18] Y. Li, P. Gegenwart, and A. A. Tsirlin, J. Phys.: Condens. Matter 32, 224004 (2020).

[19] Y. Li, H. Liao, Z. Zhang, S. Li, F. Jin, L. Ling, L. Zhang, Y. Zou, L. Pi, Z. Yang, J. Wang, Z. Wu, and Q. Zhang, Sci. Rep. 5, 16419 (2015).

[20] Y. Li, G. Chen, W. Tong, L. Pi, J. Liu, Z. Yang, X. Wang, and Q. Zhang, Phys. Rev. Lett. 115, 167203 (2015).

[21] W. Liu, Z. Zhang, J. Ji, Y. Liu, J. Li, X. Wang, H. Lei, G. Chen, and Q. Zhang, Chinese Phys. Lett. 35, 117501 (2018).

[22] J. A. M. Paddison, M. Daum, Z. Dun, G. Ehlers, Y. Liu, M. B. Stone, H. Zhou, and M. Mourigal, Nat. Phys. 13, 117 (2017).

[23] Y. Shirata, H. Tanaka, A. Matsuo, and K. Kindo, Phys. Rev. Lett. 108, 057205 (2012).

[24] T. Susuki, N. Kurita, T. Tanaka, H. Nojiri, A. Matsuo, K. Kindo, and H. Tanaka, Phys. Rev. Lett. 110, 267201 (2013).

[25] G. Koutroulakis, T. Zhou, Y. Kamiya, J. D. Thompson, H. D. Zhou, C. D. Batista, and S. E. Brown, Phys. Rev. B 91, 024410 (2015). 
[26] R. Zhong, T. Gao, N. P. Ong, and R. J. Cava, Sci. Adv. 6, eaay6953 (2020).

[27] R. Zhong, S. Guo, G. Xu, Z. Xu, and R. J. Cava, Proc. Natl. Acad. Sci. USA 116, 14505 (2019).

[28] N. Li, Q. Huang, X. Y. Yue, W. J. Chu, Q. Chen, E. S. Choi, X. Zhao, H. D. Zhou, and X. F. Sun, Nat. Commun. 11, 4216 (2020).

[29] S. Lee, C. H. Lee, A. Berlie, A. D. Hillier, D. T. Adroja, R. Zhong, R. J. Cava, Z. H. Jang, and K.-Y. Choi, Phys. Rev. B 103, 024413 (2021).

[30] The $\left(\mathrm{PO}_{4}\right)$ group has the -3 charge, as in all phosphates [51]. Its charge and the electropositive nature of $\mathrm{Na}$ and $\mathrm{Ba}$ require that the cobalt in the compound must be present as $\mathrm{Co}^{2+}$.

[31] H. Liu and G. Khaliullin, Phys. Rev. B 97, 014407 (2018).

[32] H. Liu, J. Chaloupka, and G. Khaliullin, Phys. Rev. Lett. 125, 047201 (2020).

[33] A. Abragam and B. Bleaney, Electron Paramagnetic Resonance of Transition Ions, Oxford Classic Texts in the Physical Sciences (Oxford University, New York, 1970).

[34] J. Zeisner, S. A. Bräuninger, L. Opherden, R. Sarkar, D. I. Gorbunov, J. W. Krizan, T. Herrmannsdörfer, R. J. Cava, J. Wosnitza, B. Büchner, H.-H. Klauss, and V. Kataev, Phys. Rev. B 99, 155104 (2019).

[35] C. P. Poole, Electron Spin Resonance: A Comprehensive Treatise on Experimental Techniques, Dover Books on Physics (Dover, New York, 1996).

[36] C. Slichter, Principles of Magnetic Resonance, Springer Series in Solid-State Sciences (Springer-Verlag, Berlin, 1996).

[37] H. Benner and J. P. Boucher, Spin dynamics in the paramagnetic regime: NMR and EPR in two-dimensional magnets, in Magnetic Properties of Layered Transition Metal Compounds, edited by L. J. de Jongh (Springer, New York, 1990), pp. 323-378.

[38] J. P. Perdew, K. Burke, and M. Ernzerhof, Phys. Rev. Lett. 77, 3865 (1996).
[39] K. Koepernik and H. Eschrig, Phys. Rev. B 59, 1743 (1999).

[40] GGA accounts for the splittings induced by the spin-orbit coupling and the trigonal crystal field (Fig. 1). Yet, these splittings cannot be directly visualized in the band structure (Fig. 4), because their magnitude is comparable with or smaller than the intersite hopping terms.

[41] H. Eschrig and K. Koepernik, Phys. Rev. B 80, 104503 (2009).

[42] R. O. Kuzian, O. Janson, A. Savoyant, J. van den Brink, and R. Hayn, Phys. Rev. B 104, 085154 (2021).

[43] Higher-lying multiplets shown in [Fig. 1(a)] are irrelevant in the temperature and energy range of consideration.

[44] Note that three definitions of the SO coupling constant are used for cobaltates: $\zeta$ (for the one-electron picture), $\lambda$ (for the manybody picture), and $\tilde{\lambda}$ (effective parameter for the orbital angular momentum equal to unity). For $d^{7} \mathrm{Co}^{2+}, \frac{\xi}{3} \equiv \lambda \equiv \frac{2}{3} \tilde{\lambda}$.

[45] See Supplemental Material at http://link.aps.org/supplemental/ 10.1103/PhysRevB.104.L100420 for the matrix elements of the local Wannier Hamiltonian and of the nearest-neighbor hopping term, details of application of perturbation-theory expressions for magnetic exchange integrals, the dependence of the DFT $+U$-derived magnetic exchange integrals on the $U$ value, and details of the multiplet calculations by exact diagonalization.

[46] L. Giovanelli, A. Savoyant, M. Abel, F. Maccherozzi, Y. Ksari, M. Koudia, R. Hayn, F. Choueikani, E. Otero, P. Ohresser, J.-M. Themlin, S. Dhesi, and S. Clair, J. Phys. Chem. C 118, 11738 (2014).

[47] P. Koidl, Phys. Rev. B 15, 2493 (1977).

[48] R. M. MacFarlane, J. Chem. Phys. 47, 2066 (1967).

[49] R. O. Kuzian, A. M. Daré, P. Sati, and R. Hayn, Phys. Rev. B 74, 155201 (2006).

[50] M. E. Lines, Phys. Rev. 131, 546 (1963).

[51] A. F. Wells, Structural Inorganic Chemistry (Clarendon, Oxford, 1984). 